\title{
Estudio de los costes de construcción y climatización en bodegas: tipologías constructivas de la sala de barricas respecto a la cota del terreno
}

\author{
A. Tascón' ${ }^{1}$, J. Gómez ${ }^{2}$ \\ 1 Dpto. Agricultura y alimentación, Universidad de La Rioja; alberto.tascon@unirioja.es \\ 2 Faber 1900 - Estudio de Ingeniería y Arquitectura; javier@faber1900.com
}

\begin{abstract}
Resumen: El objetivo de este trabajo fue estimar el coste de construcción y el de climatización de la sala de barricas de una bodega de Rioja, considerando 3 posibles tipologías constructivas: subterránea, parcialmente subterránea o semienterrada y aérea sobre cota cero del terreno. Las dimensiones de la sala de barricas se establecieron a partir de datos estadísticos obtenidos en trabajos previos, pudiendo considerarse como representativas de las bodegas industriales de la D.O.Ca. Rioja, y se fijó su ubicación en las proximidades de la ciudad de Logroño. En el estudio se incluyeron varias soluciones constructivas habituales para los cerramientos de fachada y de cubierta. La demanda de climatización, tanto de refrigeración como de calefacción, se calculó mediante el Método de las Series Radiantes de ASHRAE, implementado en la aplicación informática Cypetherm Loads. Los costes de construcción se calcularon a partir de una base de precios de uso habitual y el programa Presto, apoyándose también en la experiencia propia en proyectos. El análisis y comparación de los resultados obtenidos ha permitido obtener información relevante para la toma de decisiones en la fase de diseño de bodegas.
\end{abstract}

Palabras clave: crianza, Rioja, eficiencia energética, diseño, proyectos

\section{Introducción}

Es bien conocido que el vino requiere unas determinadas condiciones de temperatura y humedad para su adecuado envejecimiento y conservación [1]. Además, las condiciones higrotérmicas también influyen en las pérdidas de vino por evaporación y en el desarrollo de mohos [2].

El control de la temperatura en los locales destinados a la crianza de vino implica un coste debido al consumo de energía, excepto en aquellos casos particulares en los que no se precise un sistema mecánico de climatización. La eficiencia energética de las bodegas es un aspecto de gran interés y que puede jugar un papel relevante en el grado de competitividad y en la sostenibilidad del sector a largo plazo.

Los costes de climatización de la sala de barricas variarán en función de la tipología constructiva de la bodega respecto a la cota del terreno. Tradicionalmente, las bodegas se ejecutaban subterráneas en muchas zonas productoras, para aprovechar la mayor inercia térmica de estos espacios y conseguir así una adecuada conservación del vino sin necesidad de instalar sistemas para el control de la temperatura [3]. En la actualidad se pueden encontrar bodegas aéreas, bodegas parcialmente enterradas o semisubterráneas y bodegas subterráneas [4]; cada una de estas soluciones tendrá asociada distinta demanda energética. Otro aspecto a considerar en el diseño de la sala de crianza y en su influencia sobre la rentabilidad de la bodega es el coste asociado a su construcción, que también variará según su disposición respecto a la cota del terreno. 


\section{CONGRESO IBÉRICO DE AGROINGENIERÍA \\ X CONGRESSO IBÉRICO DE AGROENGENHARIA \\ 3 - 6 septiembre 2019, Huesca - España}

Partiendo de trabajos previos [5], en el presente estudio se ha profundizado en el análisis de los costes de construcción y climatización de una sala de crianza en barricas en relación a su disposición con respecto a la cota del terreno, considerando así mismo distintas soluciones constructivas para la envolvente de la misma.

\section{Materiales y métodos}

\subsection{Casos de estudio}

Este trabajo se ha centrado en las bodegas acogidas a la Denominación de Origen Calificada Rioja. Los casos de estudio se han definido a partir de una sala de barricas de $1547 \mathrm{~m}^{2}$, que puede considerarse representativa de las bodegas industriales de Rioja, según los datos obtenidos previamente sobre el diseño y distribución en planta de bodegas de Rioja [6]. Las dimensiones elegidas fueron $36,3 \mathrm{~m} \times 43,7 \mathrm{~m}$ en planta y una altura libre de $7 \mathrm{~m}$. La ubicación seleccionada ha sido Logroño.

En el análisis se han estudiado 3 posibles tipologías constructivas: sala de barricas totalmente subterránea (cota $-7,00 \mathrm{~m}$ ), sala parcialmente subterránea (cota $-3,50 \mathrm{~m}$ ) y sala aérea sobre cota cero del terreno (cota $0,00 \mathrm{~m}$ ). Distintas soluciones para la envolvente, habituales en los proyectos de bodegas de Rioja, han sido consideradas. En la Tabla 1 se resumen los casos de estudio.

Tabla 1. Características de las salas de crianza analizadas

\begin{tabular}{cccc}
\hline Caso no $^{\mathbf{0}}$ & Cota & Cubierta & Cerramientos \\
\hline 1 & $0,00 \mathrm{~m}$ & $\mathrm{R} 1$ & $\mathrm{~W} 1$ \\
2 & $0,00 \mathrm{~m}$ & $\mathrm{R} 1$ & $\mathrm{~W} 2$ \\
3 & $0,00 \mathrm{~m}$ & $\mathrm{R} 2$ & $\mathrm{~W} 1$ \\
4 & $0,00 \mathrm{~m}$ & $\mathrm{R} 2$ & $\mathrm{~W} 2$ \\
5 & $-3,50 \mathrm{~m}$ & $\mathrm{R} 1$ & $\mathrm{~W} 2$ \\
6 & $-3,50 \mathrm{~m}$ & $\mathrm{R} 1$ & $\mathrm{~W} 1$ \\
7 & $-3,50 \mathrm{~m}$ & $\mathrm{R} 2$ & $\mathrm{~W} 2$ \\
8 & $-3,50 \mathrm{~m}$ & $\mathrm{R} 2$ & $\mathrm{~W} 3$ \\
9 & $-7,00 \mathrm{~m}$ & $\mathrm{R} 3$ & $\mathrm{~W} 3$ \\
\hline
\end{tabular}

A continuación, se describen brevemente las características de las distintas soluciones constructivas:

- R1: teja sobre doble rastrel metálico apoyada en panel sándwich con $10 \mathrm{~cm}$ de aislante de lana de roca.

- R2: teja sobre doble rastrel metálico apoyada en placa de fibrocemento y falso techo horizontal con paneles de lana de roca de $4 \mathrm{~cm}$.

- R3: firme de hormigón de $20 \mathrm{~cm}$ sobre láminas impermeabilizantes y $12 \mathrm{~cm}$ de poliestireno extruido, todo apoyado en losa de HA de $40 \mathrm{~cm}$ (esta sala de barricas enterrada estaría situada bajo una zona de circulación de vehículos).

- R4: capa de $100 \mathrm{~cm}$ de tierra, geotextil separador y lámina drenante, capas de impermeabilización y losa de HA de $40 \mathrm{~cm}$ (esta sala de barricas enterrada estaría situada bajo zona verde, ajardinamiento, etc.).

- W1: panel de fachada prefabricado de hormigón de $24 \mathrm{~cm}$ de espesor con aislamiento a base de poliestireno expandido de $12 \mathrm{~cm}$.

- W2: fábrica de bloques de termoarcilla de $29 \mathrm{~cm}$ de espesor enfoscada con mortero de cemento por ambas caras.

- W3: muro de hormigón armado ejecutado in situ. 


\section{CONGRESO IBÉRICO DE AGROINGENIERÍA \\ X CONGRESSO IBÉRICO DE AGROENGENHARIA \\ 3 - 6 septiembre 2019, Huesca - España}

En el caso de las bodegas semienterradas, los cerramientos se componen de dos partes: la parte enterrada se resuelve mediante un muro de HA (solución W3) y la parte aérea mediante el cerramiento que corresponda, W1 o W2. La solera, en todos los casos, se ha considerado constituida por una capa de encachado de grava, una capa de hormigón y acabado superficial mediante resina epoxi.

\subsection{Demanda energética}

La demanda de climatización, tanto de refrigeración como de calefacción, de cada uno de los casos de estudio se ha calculado mediante el Método de las Series Radiantes de ASHRAE [7], implementado en el módulo Cypetherm Loads de la aplicación informática CYPE [8]. Para ello, previamente se elaboró un modelo BIM para cada una de las 3 disposiciones constructivas (bodega aérea, semisubterránea y subterránea) utilizando la aplicación IFC-Builder de Cype.

Las características de los cerramientos (conductividad térmica, densidad y calor específico) han sido obtenidos del catálogo de elementos constructivos del Código Técnico de la Edificación [9] o directamente de catálogo de fabricantes, como en el caso de los paneles prefabricados de hormigón. Además, para que las simulaciones tuvieran en cuenta la gran inercia térmica que suponen las barricas de vino almacenadas en la sala de crianza, se crearon en los modelos BIM unos espacios ficticios cerrados con tabiquería consistente en dos capas, con las propiedades térmicas de la madera de roble y del vino, de manera que el volumen de madera y líquido y el volumen total ocupado por dichos espacios fueran similares al de las pilas de barricas almacenadas en la sala (ver Figura 1); este planteamiento es similar al adoptado previamente por Benni et al. [10].

Para las simulaciones energéticas se fijaron unos intervalos de temperatura y humedad deseables de $10-18{ }^{\circ} \mathrm{C}$ y $70-85 \%$, respectivamente, y se tomaron los datos climáticos de la estación meteorológica de Agoncillo, que es la más cercana a Logroño. En cuanto a las cargas térmicas, se consideró una renovación de aire al día, una ocupación de 2 personas durante $8 \mathrm{~h} /$ día y una carga por iluminación de $6,20 \mathrm{~W} / \mathrm{m}^{2}$ durante $8 \mathrm{~h} /$ día.

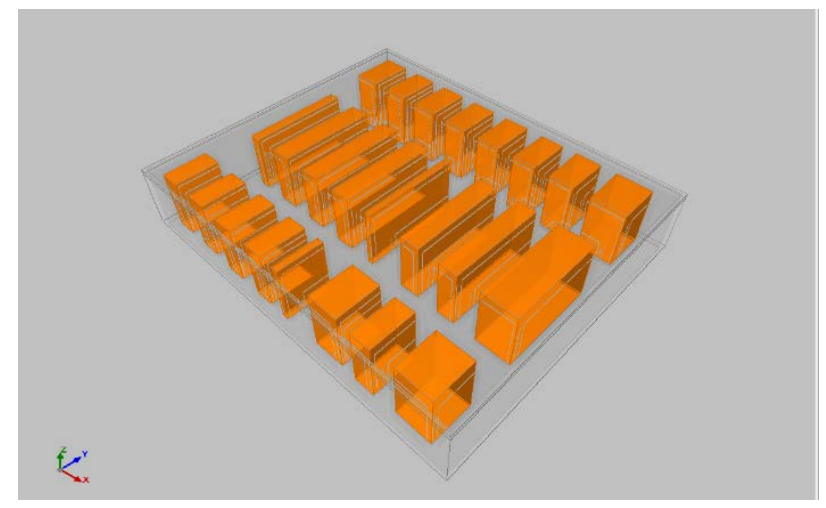

Figura 1. Modelo BIM de la sala de crianza con los espacios que simulan las pilas de barricas resaltados en color naranja.

\subsection{Coste de construcción}

Los costes de construcción han sido calculados tomando como referencia la base de precios PREOC [11] y con el apoyo de la aplicación informática Presto. Únicamente se han considerado las partidas relativas al coste de excavación, en su caso, y al de ejecución de la envolvente. El coste se ha expresado en forma de presupuesto de ejecución material. No se han incluido aquellas partidas y capítulos constructivos comunes a todas las bodegas y que son independientes de las 


\section{CONGRESO IBÉRICO DE AGROINGENIERÍA \\ X CONGRESSO IBÉRICO DE AGROENGENHARIA \\ 3 - 6 septiembre 2019, Huesca - España}

variables constructivas que se están analizando aquí, como por ejemplo fontanería, saneamiento, electricidad, incendios, pinturas y acabados, etc.

\section{Resultados y discusión}

En la Tabla 2 se presentan los resultados obtenidos en relación a la demanda energética anual. Se puede observar que las soluciones más eficientes serían las salas de crianza enterradas, luego las semienterradas y, por último, las aéreas. El caso con menor demanda energética es el caso no 9; también se indica en la Tabla 2 el incremento (\%) de la demanda energética total en relación con dicho caso.

La Tabla 3 presenta el presupuesto de ejecución material de los distintos casos de estudio considerando el movimiento de tierras, la ejecución de la envolvente (fachadas y cubierta) y, en su caso, los muros sótano incluyendo su impermeabilización y drenaje. Los resultados indican que el coste constructivo más reducido corresponde a los casos no 3 y no 4 .

Tabla 2. Demanda energética anual de calefacción $(\mathrm{C})$, refrigeración $(\mathrm{R})$ y total para las salas de crianza analizadas

\begin{tabular}{ccccccc}
\hline Caso n $^{\mathbf{0}}$ & Cota & Envolvente & $\mathbf{C} \mathbf{( k W \cdot h )}$ & R $\mathbf{( k W \cdot h )}$ & Total $\mathbf{( k W \cdot h )}$ & $\Delta(\mathbf{\%})$ \\
\hline 1 & $0,00 \mathrm{~m}$ & R1W1 & 30.811 & 163.409 & 194.221 & 83 \\
2 & $0,00 \mathrm{~m}$ & R1W2 & 27.532 & 147.378 & 174.910 & 65 \\
3 & $0,00 \mathrm{~m}$ & R2W1 & 31.519 & 168.816 & 200.335 & 89 \\
4 & $0,00 \mathrm{~m}$ & R2W2 & 33.663 & 147.161 & 180.824 & 70 \\
5 & $-3,50 \mathrm{~m}$ & R1W1 & 23.795 & 146.583 & 170.378 & 60 \\
6 & $-3,50 \mathrm{~m}$ & R1W2 & 22.132 & 137.532 & 159.664 & 50 \\
7 & $-3,50 \mathrm{~m}$ & R2W1 & 27.162 & 151.048 & 178.210 & 68 \\
8 & $-3,50 \mathrm{~m}$ & R2W2 & 25.805 & 142.892 & 168.697 & 59 \\
9 & $-7,00 \mathrm{~m}$ & R3W3 & 20.620 & 85.603 & 106.223 & 0 \\
10 & $-7,00 \mathrm{~m}$ & R4W3 & 23.539 & 95.104 & 118.643 & 12 \\
\hline
\end{tabular}

La demanda energética anual presentada en la Tabla 2 da una indicación sobre el coste energético asociado a cada solución de diseño. Si se consideran las instalaciones concretas y fuentes de energía con las que se cubrirá dicha demanda energética (equipos mecánicos con consumo de energía eléctrica, bombas de calor con apoyo geotérmico o solar, biomasa, etc.), se podría determinar el coste anual en euros asociado a dicha demanda; esa es una línea de trabajo que servirá para complementar y ampliar el presente estudio. Con ello, se podrían comparar en euros los costes energéticos y de construcción para la toma de decisiones en la fase de diseño de un proyecto.

Tabla 3. Coste de construcción de las salas de crianza analizadas

\begin{tabular}{ccccc}
\hline Caso $\mathbf{~ n}^{\mathbf{0}}$ & Cota & Envolvente & Coste $(\boldsymbol{€})$ & $\Delta(\mathbf{\%})$ \\
\hline 1 & $0,00 \mathrm{~m}$ & R1W1 & $214.330,51$ & 24 \\
2 & $0,00 \mathrm{~m}$ & R1W2 & $219.985,80$ & 27 \\
3 & $0,00 \mathrm{~m}$ & R2W1 & $173.305,51$ & 0 \\
4 & $0,00 \mathrm{~m}$ & R2W2 & $178.960,80$ & 3 \\
5 & $-3,50 \mathrm{~m}$ & R1W1 & $309.513,59$ & 79 \\
6 & $-3,50 \mathrm{~m}$ & R1W2 & $312.341,24$ & 80 \\
7 & $-3,50 \mathrm{~m}$ & R2W1 & $268.488,59$ & 55 \\
8 & $-3,50 \mathrm{~m}$ & R2W2 & $271.316,24$ & 57 \\
9 & $-7,00 \mathrm{~m}$ & R3W3 & $693.749,12$ & 300 \\
10 & $-7,00 \mathrm{~m}$ & R4W3 & $615.109,47$ & 255 \\
\hline
\end{tabular}




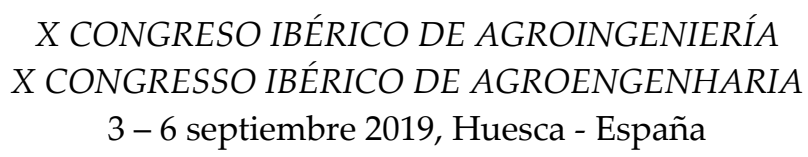

\section{Conclusiones}

El presente estudio ha permitido obtener información sobre el coste de construcción y la demanda energética de climatización de salas de barricas aéreas, semienterradas y subterráneas, considerando varias soluciones constructivas habituales para los distintos elementos de la envolvente del edificio. La metodología de estudio utilizada puede ser fácilmente aplicada en proyectos reales, por lo que puede ser empleada para la selección de alternativas de proyecto y la toma de decisiones de diseño. De los resultados obtenidos se puede concluir que la cota seleccionada para la ejecución de la bodega tiene una influencia significativa, tanto en la demanda energética como en los costes de ejecución. Como línea de trabajo para continuar el presente estudio, la selección de instalaciones y equipos energéticos concretos permitiría calcular el coste anual asociado a la demanda energética y así poder comparar y seleccionar el diseño más óptimo para cada situación.

\section{Agradecimientos}

Este trabajo ha contado con financiación del Instituto de Estudios Riojanos mediante el proyecto Análisis de los costes de construcción y climatización en las bodegas de Rioja: optimización de tipologías constructivas.

\section{Referencias}

1. Hidalgo Togores J. Tratado de Enología. Ed. Mundi-Prensa, 2003.

2. Blouin J., Maron J.M. Control de las temperaturas y calidad de los vinos. Ed. Acribia, 2008.

3. Mazarrón F.R., López-Ocón E., Garcimartín M.A., Cañas I. Assessment of basement constructions in the winery industry. Tunnelling and underground space technology. 2013, vol. 35, 200-206.

4. Yravedra Soriano M.J. Arquitectura y cultura del vino. Ed. Munilla-Lería, 2003.

5. Gómez J., Ruiz de Adana M.M., Altuzarra A. Análisis de costes de implantación y climatización de bodegas de crianza aéreas, semienterradas y enterradas. VI Foro Mundial del Vino, Logroño, España, 2008. Libro de Actas, CD-ROM, ISBN: 978-84-8125-306-1.

6. Gómez J., Tascón A., Ayuga F. Systematic layout planning of wineries: the case of Rioja region (Spain). Journal of Agricultural Engineering. 2018, vol. 49, 34-41.

7. ASHRAE Handbook, Vol. 1: Fundamentals. American Society of Heating, Refrigerating and AirConditioning Engineers, 2017.

8. CYPETHERM LOADS. Carga térmica de los edificios. CYPE Ingenieros. En Internet: $<$ http://cypetherm-loads.cype.es/>

9. Instituto Eduardo Torroja de ciencias de la construcción. Catálogo de elementos constructivos del CTE. Ministerio de Vivienda, 2009.

10. Benni S., Torreggiani D., Barbaresi A., Tassinari P. Thermal performance assessment for energyefficient design of farm wineries. Transactions of the ASABE. 2013, vol. 56, 1483-1491.

11. PREOC 2018. Base de precios de edificación y obra civil. Atayo, 2018. En internet: $<$ http://www.preoc.es> 